\title{
Analytical Modeling and Simulation of Four-Switch Hybrid Power Filter Working with Sixfold Switching Symmetry
}

\author{
Josef Tlustý, ${ }^{1}$ Jiří Škramlík, ${ }^{2}$ Jan Švec, $^{1}$ and Viktor Valouch $^{2}$ \\ ${ }^{1}$ Department of Electrical Power Engineering, Faculty of Electrical Engineering, \\ Czech Technical University in Prague, Technická 2, 16627 Prague 6, Czech Republic \\ ${ }^{2}$ Institute of Thermomechanics, v.v.i., Academy of Sciences of the Czech Republic, \\ Dolejškova 5, 18202 Praha 8, Czech Republic \\ Correspondence should be addressed to Jan Švec, jan.svec@fel.cvut.cz
}

Received 7 March 2012; Revised 3 July 2012; Accepted 6 August 2012

Academic Editor: Stefano Lenci

Copyright (C) 2012 Josef Tlustý et al. This is an open access article distributed under the Creative Commons Attribution License, which permits unrestricted use, distribution, and reproduction in any medium, provided the original work is properly cited.

\begin{abstract}
Shunt active power filters are nowadays used for mitigation of harmonic currents from nonlinear loads. Current and voltage harmonics have negative effects on the operation of the electric power system. Thus, a great attention is focused on harmonic generation and control. Several standards have introduced limits on current harmonics injected into the power system and on voltage harmonics at the system busbars. The paper proposes a switching strategy for a fourswitch converter as used in conventional six-switch converters. The voltage source converter is controlled by a unique space-vector modulation strategy proposed. Here is presented an analytical solution for a proposed active power filter topology, simulation, and experiments results proving the proposed strategy correctness and its characteristics.
\end{abstract}

\section{Introduction}

Nowadays, more and more nonlinear loads generate current and voltage harmonics that have negative effects on the operation of electric power systems.

A conventional measure for the compensation of nonsinusoidal currents and/or lagging load power factor is represented by tuned parallel passive filters (PFs). However, their frequency characteristics are unfavorably influenced by the variable grid impedance and the variations of the filter parameters which occur due to aging, temperature, and other operational conditions. 
Active power filters (APFs) made up of a power converter have been developed to suppress the harmonic currents and improve power factor.

Hybrid power filters (HPFs) consisting of dedicated passive and active filters connected in series or parallel have been developed. The tolerances and variations in the hybrid filter are not as serious as those in a passive filter used alone. It is well known that HPF helps to reduce the active filter rating [1].

The parallel APF provides very good filter characteristics, but the VA capacity of the power converter is the product of the load current harmonic plus fundamental reactive components and the power grid voltage. Thus, the capacity and manufacture cost of the power converter are very high which limit the APF wide application.

The HPF consists of a PF and a power converter. In the HPF operation, the PF can reduce the capacity of the power converter, whereas the power converter is used to improve the PF filter characteristic. So the HPFs are suitable especially for high-power nonlinear loads.

Although many modern power semiconductor devices offer a high degree of reliability, a three-phase converter can develop various faults because of unexpected load conditions or erroneous operation.

These faults have been analyzed and remedial strategies suggested, for example, in $[2,3]$. The converter turning off is not possible in many applications from the point of view of technology requirements. Some solutions have been suggested for this situation, commonly leading to the controller reconfiguration.

The three-phase converter with only two converter-legs (B4) connection, where one leg is connected directly with the dc bus voltage center, is an alternative solution [4]. Only four power switches and four clamping diodes are used in the adopted converter instead of six power switches and six clamping diodes used in a conventional converter (B6) connection. But the main disadvantages of four-switch presented topology are as follows.

(i) The dc-bus voltage center must be accessible as one of the three converter phases is directly connected with this center point.

(ii) The dc-link capacitor size must be high to prevent a pulsation of the dc-bus center point as the current going through this point is not dc, but it has ac character.

In, [5] we investigated a four-switch APF space vector modulated with the fourfold symmetry. In [6], we applied the same fourfold symmetry to the HPF. This switching symmetry is different from a sixfold switching symmetry of a classical six-switch converter.

In this paper, we propose the same switching symmetry (sixfold) as in a normal (B6) converter for a four-switch converter shown in Figure 1. The voltage source converter is controlled by a unique space-vector modulation (SVM) strategy proposed for B4 connection. It means that all switching times valid for one-sixth of the output period are the same also in the remaining sixths of the output period. For the analytical investigation of such a system we used the mixed $p-z$ approach [7] to analyze the SVM-suggested modification for the component-minimized voltage source converter (VSC). First, unsymmetrical voltage spacevectors are decomposed into symmetrical components using discrete Fourier transform (DFT). Then, for the voltage symmetrical components, the mathematical model makes use of the Laplace and modified Z-transform to predict responses of the line currents. 


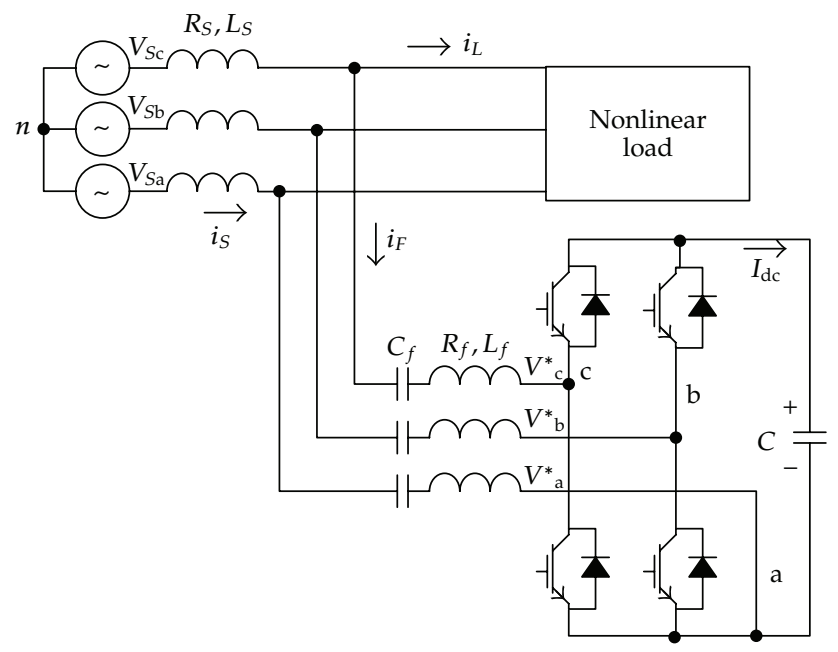

Figure 1: Configuration of the proposed topology.

\section{System Configuration}

In this paper, a new topology introduced in [1] is analytically investigated and simulated. The system configuration is shown in Figure 1. The passive part consists of a series inductor and capacitor set (resistor, inductance, and capacitor: $R_{F}, L_{F}$, and $C_{F}$ ), and the VSC is a two-leg bridge structure. As it can be seen in this configuration, one phase (in our example phase "a") of the passive filter is directly connected with one of the four-switch converter dc terminals (in our example minus dc bus terminal). Consequently, the number of power-switching devices is reduced, and disadvantages (i)-(ii) are avoided as the dc midbus point is not utilized. The voltage-source power converter with only two arms is controlled by the space-vector sixfold pulse width modulation strategy.

\section{Space Vector Modulation for B4 Connection with Sixfold Symmetry}

Voltage source vectors for B6 converter are depicted in Figure 2. Since the phase "a" is connected with the negative dc bus terminal in our new topology, we can use only 4 vectors shown in the shade area $\left(\mathbf{V}_{0}, \mathbf{V}_{3}, \mathbf{V}_{4}\right.$, and $\left.\mathbf{V}_{5}\right)$. But each phase of the proposed HPF contains a capacitor which can block the dc component of the power converter's output. Thus, we can see that this space-vector diagram contains a dc offset voltage in each of three phases.

Let the vectors $\left(\mathbf{V}_{0}, \mathbf{V}_{3}, \mathbf{V}_{4}\right.$, and $\left.\mathbf{V}_{5}\right)$ in Figure 2 have the following denotation:

$$
\mathbf{V}(Z 00), \mathbf{V}(Z 10), \mathbf{V}(Z 11), \mathbf{V}(Z 01),
$$

where $\left(Z, S_{b}, S_{c}\right)$ in (3.1) denotes the inverter switching states where $S_{i}=1(i=b, c)$ if the upper leg switch is on and $S_{i}=0(i=b, c)$ if the upper leg switch is off. $\mathrm{Z}$ means zero 


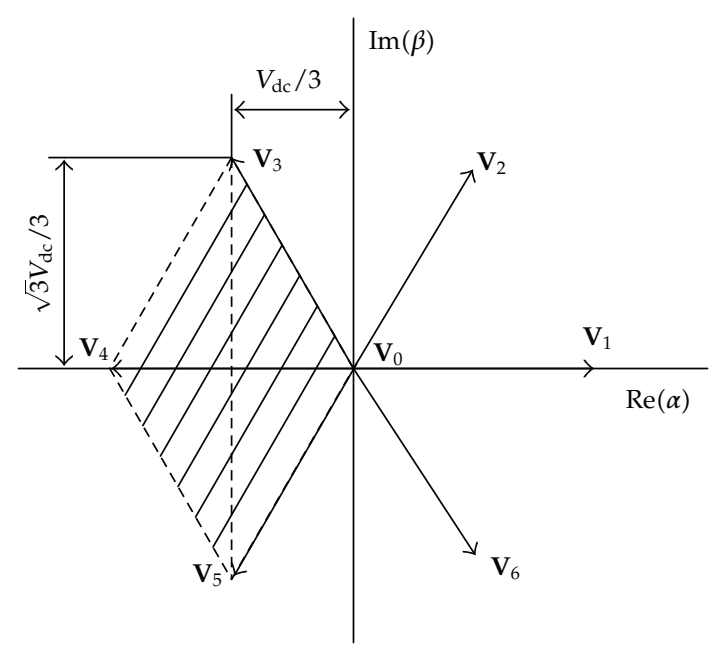

Figure 2: Voltage space vectors in proposed converter.

pole voltage $V_{\mathrm{A}}=0$. It means that from the ac utility point of view, the voltage space vector diagram in Figure 2 is shifted by a dc-offset voltage space vector with the magnitude

$$
\mathbf{V}_{\text {off }}=\frac{-V_{\mathrm{dc}}}{3}
$$

As it can be seen from Figure 2, the capacitors dc-phase components forming the voltage space vector $\mathbf{V}_{\text {off }}$ can be derived as $V_{\mathrm{fa} 0}=-V_{\mathrm{dc}} / 3, V_{\mathrm{fb} 0}=V_{\mathrm{fc} 0}=V_{\mathrm{dc}} / 6$.

Therefore, the offset $0.5 V_{\mathrm{dc}}$ in the phase voltages at converter terminals resulting from the negative bus connection of the phase "a" is compensated by this voltage space vector $\mathbf{V}_{\text {off. }}$.

Since the dc bus voltage of the HPF power converter is relatively small as compared to that of a parallel APF, only a small additional voltage rating, resulting from the presence of the voltage space vector $\mathbf{V}_{\text {off }}$, is required for the capacitors of this passive part of the HPF.

Thus, we have four switching states in B4 configuration. Four possible voltage space vectors $\mathbf{V}(\mathrm{Z} 00), \mathbf{V}(\mathrm{Z} 10), \mathbf{V}(\mathrm{Z} 11)$, and $\mathbf{V}(\mathrm{Z} 01)$ in the complex $\alpha \beta$ plane can be found as shown in Figure 3. These vectors are $\pi / 2$ away from each other. But they do not have the same amplitude as in the classical B6 connection. Vectors laying in the real axis have the amplitude of $V_{\mathrm{dc}} / 3$ whereas the vectors lying in the imaginary axis have the amplitude of $\sqrt{3} V_{\mathrm{dc}} / 3$.

As mentioned earlier for this connection with only four switches, we want to have the same switching symmetry as in normal B6 converter. To form sixfold symmetry, we must use some vectors twice for the whole output period. Instead of system (3.1), we can use the system

$$
\mathbf{V}(Z 00), \mathbf{V}(Z 00), \mathbf{V}(Z 10), \mathbf{V}(Z 11), \mathbf{V}(Z 11), \mathbf{V}(Z 01)
$$




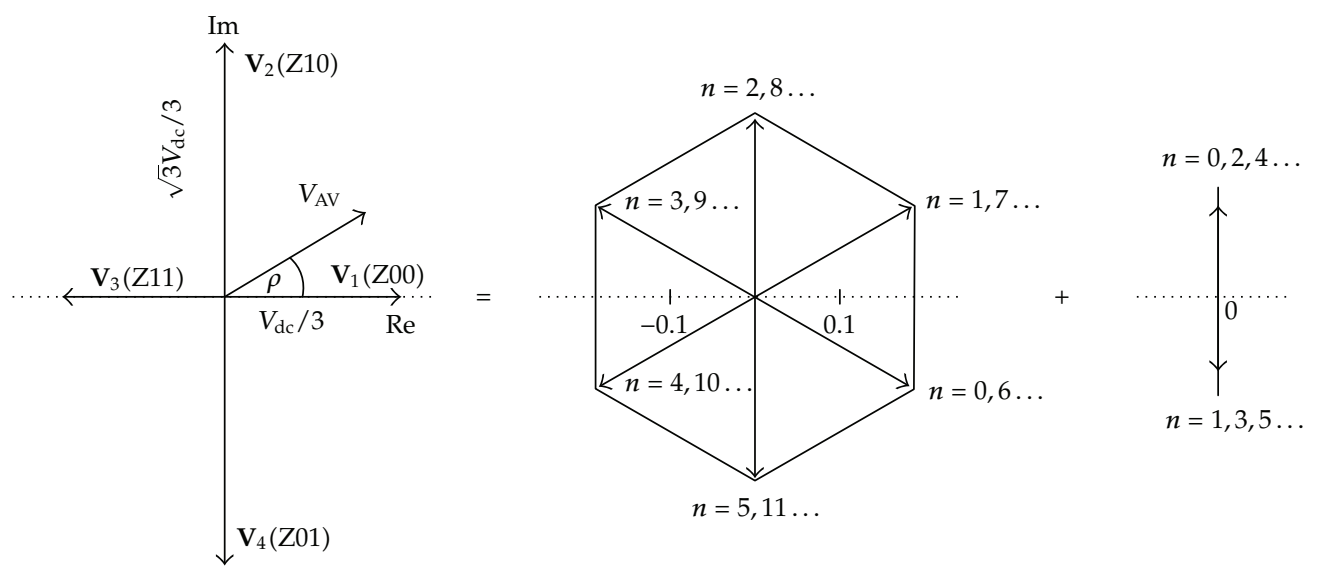

Figure 3: Voltage space vector decomposition.

For the next calculations, we express the time in terms of the sector number $n$ and per unit time variable $\varepsilon$ as

$$
t=\frac{(n+\varepsilon) T_{1}}{6}=(n+\varepsilon) T, \quad n=0,1,2, \ldots, 0 \leq \varepsilon \geq 1,
$$

$T_{1}$ is the fundamental period, $T$ is the periodicity time of a space vector modulation, and it is called a sector period

$$
T=\frac{T_{1}}{6} .
$$

Using discrete Fourier Transform (DFT) [8], the unsymmetrical sequence (3.3) can be decomposed into two symmetrical ones as follows:

$$
\mathbf{V}(n)=\frac{V_{\mathrm{dc}}}{3} \frac{2}{\sqrt{3}} e^{-\mathrm{j} \pi / 6} e^{\mathrm{j} \pi(n / 3)}+\frac{V_{\mathrm{dc}}}{3} \frac{1}{\sqrt{3}} e^{\mathrm{j} \pi / 2} e^{\mathrm{j} \pi n}=\mathbf{V}^{(1)}(n)+\mathbf{V}^{(3)}(n) .
$$

As it can be seen from (3.6), the unsymmetrical vector sequence (3.3) can be decomposed into two symmetrical ones. The first sequence has a symmetry $\pi / 3$ as in the conventional B6 inverter, but its amplitude is lowered $1 / \sqrt{3}$ times, and the phase is rotated by $(-\pi / 6)$ as compared with the conventional B6 converter.

The second symmetrical sequence is laying in the imaginary axis, and it has the amplitude lowered twice compared with the first sequence. This sequence will form the thirdorder voltage harmonics in the output cycle period.

Now such a modulation strategy has to be proposed that has the same symmetry as for the B6 converter and is simple without significant increase in switching frequency. The proposed modulation strategy is realized by planning the switching patterns between the four active voltage vectors within a sampling period.

(a) Utilizing the first voltage vector sequence term $\mathbf{V}^{(1)}(n)$ in (3.6). As in a usual space vector modulation (SVM), four consecutive voltage vectors in a sampling 
period are used to generate the average output voltage matching with the reference voltage.

(b) Minimizing the second voltage vector sequence term $\mathbf{V}^{(3)}(n)$ in (3.6). For minimization, the average voltage from the $\mathbf{V}^{(3)}(n)$ within a sampling period should be zero. Thus, the resulting converter fundamental voltage is balanced under steady states although the sequence (3.3) represents an unbalanced fundamental voltage. Nevertheless, some small residual third-order voltage harmonics in the output cycle period are generated. It will be seen in the simulation as well as experimental results presented later.

The converter should generate an average voltage vector $\mathbf{V}_{\mathrm{AV}}$ within each of the sampling periods. Unlike the conventional SVM techniques, here four adjacent voltage vectors will be used in every sampling period to provide us with an approximation of the demanded vector $\mathbf{V}_{\mathrm{AV}}$ in this period.

Let every sixth of the fundamental period of the length $T_{1}$ be divided into $N_{1}$ segments with a sampling period

$$
\Delta T=\frac{T_{1}}{6 N_{1}}=\frac{T}{N_{1}} .
$$

Thus, the following three equations should be satisfied in order that four applied voltage vectors may generate an average converter voltage vector matching the vector $\mathbf{V}_{\mathrm{AV}}$ and the conditions (a) and (b) as well:

$$
\begin{gathered}
\mathbf{V}_{\mathrm{AV}} e^{j \rho} \Delta T=\frac{2 V_{\mathrm{dc}}}{3 \sqrt{3}} \Delta T_{1} e^{-j \pi / 6}+\frac{2 V_{\mathrm{dc}}}{3 \sqrt{3}} \Delta T_{2} e^{j \pi / 6}+\frac{2 V_{\mathrm{dc}}}{3 \sqrt{3}} \Delta T_{3} e^{j \pi / 2}+\frac{2 V_{\mathrm{dc}}}{3 \sqrt{3}} \Delta T_{4} e^{j 5 \pi / 6}, \\
\Delta T_{1}+\Delta T_{3}=\Delta T_{2}+\Delta T_{4} \\
\Delta T=\Delta T_{1}+\Delta T_{2}+\Delta T_{3}+\Delta T_{4}
\end{gathered}
$$

where $\rho$ is the angle to the real axis, and $\Delta T_{1}, \Delta T_{2}, \Delta T_{3}$, and $\Delta T_{4}$ are the time portions allocated to the vectors $\mathbf{V}(Z 00), \mathbf{V}(Z 10), \mathbf{V}(Z 11)$, and $\mathbf{V}(Z 01)$. Solving (3.8), we obtain the following switching time intervals (in p.u. time related to the time interval $\Delta T$ of the sixth of the period):

$$
\begin{aligned}
& \Delta \varepsilon_{1}=\frac{\Delta T_{1}}{T}=-\frac{g}{2 N_{1}} \sin (\rho)+\frac{1}{2 N_{1}}, \\
& \Delta \varepsilon_{2}=\frac{\Delta T_{2}}{T}=\frac{g}{2 N_{1}} \sin \left(\rho+\frac{\pi}{3}\right), \\
& \Delta \varepsilon_{3}=\frac{\Delta T_{3}}{T}=\frac{g}{2 N_{1}} \sin (\rho), \\
& \Delta \varepsilon_{4}=\frac{\Delta T_{4}}{T}=\frac{1}{N_{1}}-\Delta \varepsilon_{1}-\Delta \varepsilon_{2}-\Delta \varepsilon_{3},
\end{aligned}
$$


where

$$
g=\frac{2 \sqrt{3} \mathbf{V}_{\mathrm{AV}}}{V_{\mathrm{dc}}}
$$

is the modulation index.

Since the four-switch converter is operated with the SVM strategy described here, expression (3.6) must be modified to respect the switching intervals (3.9) as follows:

$$
\begin{aligned}
\mathbf{V}(n, \varepsilon) & =\mathbf{V}^{(1)}(n, \varepsilon)+\mathbf{V}^{(3)}(n, \varepsilon) \\
& =\sum_{k=1}^{4 N_{1}} V_{\mathrm{dc}} \frac{2}{3 \sqrt{3}} e^{j(n-1 / 2) \pi / 3} f(\varepsilon, k) e^{j(\pi / 3) \alpha(k)}+\sum_{k=1}^{4 N_{1}} V_{\mathrm{dc}} \frac{1}{3 \sqrt{3}} e^{j(n+1 / 2) \pi} f(\varepsilon, k) e^{j \pi \alpha(k)} .
\end{aligned}
$$

Four adjacent vectors used in the SVM strategy are characterized by

$$
\alpha(k)=(k \text { modulo } 4)-1
$$

while the switching functions $f(\varepsilon, k)$ describing the switching patterns are defined as follows:

$$
\begin{aligned}
& f(\varepsilon, k)=1 \quad \text { if } \varepsilon_{A k} \leq \varepsilon \leq \varepsilon_{B k} \\
& f(\varepsilon, k)=0 \ldots \text { else }
\end{aligned}
$$

where $\varepsilon_{A k}, \varepsilon_{B k}$ are the start point setting time and the end point setting time, respectively, of the $k$ th voltage vector. The switching time intervals $\varepsilon_{B k}-\varepsilon_{A k}=\Delta \varepsilon_{k}$ (p.u.) are defined by (3.9).

\section{Analytical Solution in the Closed Form}

We can write the voltage equations for the circuit in Figure 1 in the space vector notation (in the Laplace transform)

$$
\begin{gathered}
\mathbf{v}_{\mathrm{S}}(s)=\left(R_{\mathrm{S}}+s L_{\mathrm{S}}\right) \mathbf{i}_{\mathrm{S}}(s)+\left(R_{\mathrm{F}}+s L_{\mathrm{F}}+\frac{1}{s C_{\mathrm{F}}}\right) \mathbf{i}_{\mathrm{F}}(s)+\mathbf{v}^{*}(s), \\
\mathbf{i}_{S}(s)=\mathbf{i}_{L}(s)+\mathbf{i}_{\mathrm{F}}(s)
\end{gathered}
$$

where $\mathbf{v}^{*}(s)$ is the Laplace transform of the VSC output voltage [6].

To find the Laplace transform of the converter ac terminal voltage vector $\mathbf{v}^{*}(s)$, we can use the Laplace transform of the periodic voltage vector as follows:

$$
\mathbf{v}^{*}(s)=\sum_{n=0}^{\infty} \int_{0}^{1} \mathbf{V}(n, \varepsilon) e^{-s(n+\varepsilon) T} T d \varepsilon=T \int_{0}^{1} \mathbf{V}(z, \varepsilon) \cdot z^{-\varepsilon} d \varepsilon,
$$


where $z=e^{s T}$, and $\mathbf{V}(z, \varepsilon)$ is the modified Z-transform of $\mathbf{V}(n, \varepsilon)[4]$, defined as follows:

$$
\mathbf{V}(z, \varepsilon)=\sum_{n=0}^{\infty} \mathbf{V}(n, \varepsilon) z^{-n}
$$

By the modified Z-transform, we can cover discrete system not only in the sampling times $t$ $=n T$ as for normal $Z$-transform but also at all instants of time using $t=n T+\varepsilon T$.

Thus, the voltage vector $\mathbf{v}^{*}(s)$ is as follows:

$$
\begin{aligned}
\mathbf{v}^{*}=\mathbf{V}^{(1)}(s)+\mathbf{V}^{(3)}(s)= & \frac{1}{s} \frac{2 V_{\mathrm{dc}}}{3 \sqrt{3}}\left[\frac{e^{s T} e^{-j \pi / 6}}{e^{s T}-e^{j \pi / 3}} \sum_{k=1}^{M} e^{j \pi \alpha(k) / 3}\left(e^{-s T \varepsilon_{A k}}-e^{-s T \varepsilon_{B k}}\right)\right] \\
& +\frac{1}{s} \frac{V_{\mathrm{dc}}}{3 \sqrt{3}}\left[\frac{e^{s T} e^{j \pi / 2}}{e^{s T}-e^{j \pi}} \sum_{k=1}^{M} e^{j \pi \alpha(k)}\left(e^{-s T \varepsilon_{A k}}-e^{-s T \varepsilon_{B k}}\right)\right],
\end{aligned}
$$

where $M=4 N_{1}$ is the number of vectors that are used within the sector period $T ; \varepsilon_{A k}, \varepsilon_{B k}$ are, respectively, the beginning and the end of $k$ th voltage space vector application inside a sector period. These times are given by switching SVM strategy described above.

As we assume that a grid voltage vector contains the positive and negative parts, its Laplace transform is given as follows:

$$
\mathbf{v}_{S}(s)=\frac{V_{\mathrm{SP}} e^{j \varphi_{P}}}{s-j \omega_{1}}-\frac{V_{\mathrm{SN}} e^{j \varphi_{N}}}{s+j \omega_{1}}
$$

where $V_{\mathrm{SP}}, e^{j \varphi_{P}}$ and $V_{\mathrm{SN}}, e^{j \varphi_{N}}$ are, respectively, the amplitude and phase angle of the positive and negative components of the grid voltage vector.

As a nonlinear load, we assume a full bridge rectifier. This load current space vector can be expressed as

$$
\mathbf{i}_{L}(n, \varepsilon)=\frac{2}{\sqrt{3}} I_{d} e^{j \pi / 2} e^{j \pi(n-1) / 3},
$$

with the phase load current given by a real part of (4.6) and shown in Figure 4 ( $I_{d}$ is a dc current of the full bridge rectifier).

The Laplace transform of (4.6) may be found again by using (4.2)-(4.3)

$$
\mathbf{i}_{L}(s)=\frac{2}{\sqrt{3}} I_{d} \frac{1}{s} \frac{e^{s T} e^{j \pi / 6}}{e^{s T}-e^{j \pi / 3}}\left(1-e^{-s T}\right)
$$

For calculation of the switching times by means of (3.9), we need to know the modulation index $g$ and the polar angle $\rho$ of the reference voltage $\mathbf{V}_{\mathrm{AV}}$ to the real axis. These parameters are not constants as in the classical SVM because of using here the two converter-legs (B4) connection that generates compensating harmonics. The parameters may be calculated as follows. 


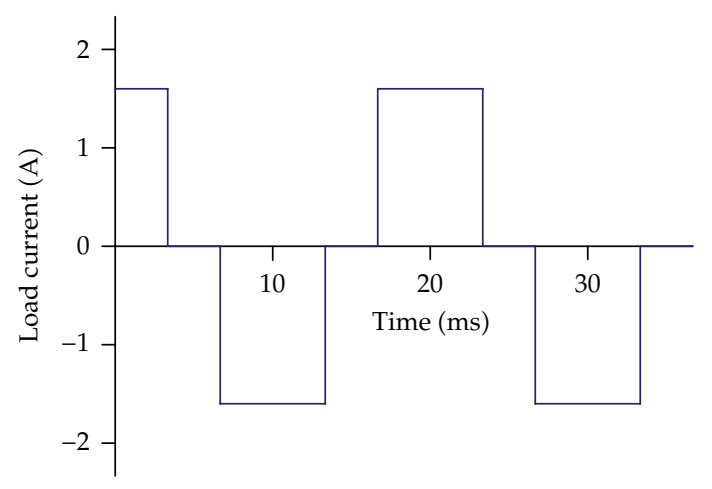

Figure 4: Nonlinear load current.

For the steady state, we can write for the VSC terminal voltage, grid, and VSC currents

$$
\begin{aligned}
& \mathbf{V}_{S}=V_{\mathrm{SP}} e^{j \omega_{1} t}+V_{\mathrm{SN}} e^{-j \omega_{1} t} e^{j \gamma}, \\
& \mathbf{I}_{S}=I_{\mathrm{SP}} e^{j \omega_{1} t} e^{j \varphi}, \\
& \mathbf{I}_{F}=I_{F 1} e^{j \omega_{1} t} e^{j \gamma_{F 1}}+\sum_{h=3,-5,7, \ldots} \alpha_{h} I_{L h} e^{j \omega_{h} t}, \\
& \mathbf{V}_{\mathrm{AV}}=V_{\mathrm{AV}} e^{j \rho},
\end{aligned}
$$

where $\gamma$ is the angle between the positive and negative sequences of the grid voltage, $\varphi$ is the angle between $\mathbf{V}_{\mathrm{SP}}$ and $\mathbf{I}_{S}$ (for $\varphi=0$, the power factor equals 1), $\omega_{1}$ is an angular frequency of the grid voltage, and $\omega_{h}=h \omega_{1}$. The magnitude $I_{\mathrm{F} 1}$ and angle $\gamma_{F 1}$ of the fundamental converter current depend on whether the reactive load power should be compensated by the HPF or not. $\alpha_{h}(\leq 1)$ is a factor determining the expected effectiveness of harmonic current suppression by using this converter-based HPF. $\mathbf{V}_{\mathrm{AV}}$ is an average voltage vector that is equal to the actual value of the vector $\mathbf{v}^{*}$ (Figure 3 ) in the middle of the sampling period $\left(\mathbf{V}_{\mathrm{AV}}=\mathbf{v}^{*}\right)$.

Thus, for given $\mathbf{V}_{S}, \mathbf{I}_{S}, \mathbf{I}_{L}, \omega_{1}, Z_{S}$, and $Z_{F}$, we can calculate the modulation index and phase of the reference voltage vector $\mathbf{V}_{\mathrm{AV}}$,

$$
\mathbf{V}_{\mathrm{AV}}=\mathbf{V}_{S}-\mathbf{I}_{S}\left(Z_{S}+Z_{F}\right)-Z_{F} \mathbf{I}_{F}=\frac{V_{\mathrm{dc}}}{2 \sqrt{3}} g(\varepsilon) e^{j p(\varepsilon)}
$$

Now we are prepared to calculate the time response of the grid current $\mathbf{i}_{S}(n, \varepsilon)$. This current can be determined by using the mixed $p-z$ approach in the analytical closed form. follows:

The expression for this current in the Laplace domain is given on the basis of (4.1), as

$$
\mathbf{i}_{S}(s)=\frac{1}{Z_{S}(s)+Z_{F}(s)} \cdot\left(\mathbf{v}_{S}(s)+Z_{F}(s) \mathbf{i}_{L}(s)-\mathbf{v}^{*}(s)\right),
$$

where $\mathbf{v}_{s}(s), \mathbf{i}_{L}(s)$, and $\mathbf{v}^{*}(s)$ are determined by (4.5), (4.7), and (4.4). 
However, the last two terms in (4.10) cannot be made in a direct way as (4.10) contains infinite number of poles given by (see (4.4), (4.7))

$$
\begin{gathered}
e^{s T}-e^{j \pi / 3}=0, \\
e^{s T}-e^{j \pi}=0 .
\end{gathered}
$$

To find the closed-form solution of (4.10) in the time domain, we should transfer the Laplace transform of the grid currents into the modified Z-transform [7].

Thus, the current $\mathbf{i}_{S}(s)$ (4.10) can be written in the form

$$
\mathbf{i}_{S}(s)=\frac{1}{Z_{S}(s)+Z_{F}(s)} \cdot \mathbf{v}_{S}(s)+\frac{e^{s T}}{e^{s T}-e^{j \pi / 3}} Q_{L}(s)-\frac{e^{s T}}{e^{s T}-e^{j \pi / 3}} Q^{(1)}(s)-\frac{e^{s T}}{e^{s T}-e^{j \pi}} Q^{(3)}(s),
$$

where

$$
\begin{aligned}
Q_{\mathrm{L}}(s) & =\frac{2}{\sqrt{3}} I_{d} \frac{1}{S} e^{j \pi / 6}\left(1-e^{-s T}\right) \frac{Z_{F}(s)}{Z_{S}(s)+Z_{F}(s)}, \\
Q^{(1)}(s) & =\frac{1}{s} \frac{2 V_{\mathrm{dc}}}{3 \sqrt{3}} e^{-j \pi / 6} \sum_{k=1}^{M} e^{j \pi \alpha(k) / 3}\left(e^{-s T \varepsilon_{A k}}-e^{-s T \varepsilon_{B k}}\right) \frac{1}{Z_{S}(s)+Z_{F}(s)}, \\
Q^{(3)}(s) & =\frac{1}{s} \frac{V_{\mathrm{dc}}}{3 \sqrt{3}} e^{j \pi / 2} \sum_{k=1}^{M} e^{j \pi \alpha(k)}\left(e^{-s T \varepsilon_{A k}}-e^{-s T \varepsilon_{B k}}\right) \frac{1}{Z_{S}(s)+Z_{F}(s)},
\end{aligned}
$$

and the modified Z-transform of (4.12) can be expressed as

$$
\begin{aligned}
\mathbf{i}(z, \varepsilon)= & Z_{m}\left\{\frac{1}{Z_{S}(s)+Z_{F}(s)} \cdot \mathbf{v}_{S}(s)\right\}+R_{L}(z) Z_{m}\left\{Q_{L}(s)\right\} \\
& -R^{(1)}(z) Z_{m}\left\{Q^{(1)}(s)\right\}-R^{(3)}(z) Z_{m}\left\{Q^{(3)}(s)\right\},
\end{aligned}
$$

where

$$
\begin{gathered}
R_{L}(z)=R^{(1)}(z)=\frac{z}{z-e^{j \pi / 3}} \\
R^{(3)}(z)=\frac{z}{z-e^{j \pi}} .
\end{gathered}
$$

To find the modified Z-transform of $Q_{\mathrm{L}}(s), Q^{(1)}(s)$, and $Q^{(3)}(s)$ in (4.13), we can use the translation theorem that reads

$$
Z_{m}\left\{e^{-s a} F(s)\right\}=z^{-y} F(z, \varepsilon-a+y) .
$$


The parameter $y$ is given by

$$
\begin{aligned}
& y=1 \text { for } 0 \leq \varepsilon<a, \\
& y=0 \text { for } a \leq \varepsilon<1 .
\end{aligned}
$$

To express the translation for the $k$ th pulse with the beginning $\varepsilon_{A k}$ and the end $\varepsilon_{B k}$ (the pulse width is $\Delta \varepsilon_{k}=\varepsilon_{B k}-\varepsilon_{A k}$ ), we use parameters $m_{k}$ and $n_{k}$ to determine per unit time for a prepulse, inside-pulse, and postpulse. The parameter $m_{k}$ defines the beginning of the $k$ th pulse $\varepsilon_{A k}$, while the parameter $n_{k}$ defines the end of the $k$ th pulse $\varepsilon_{B k}$. According to (4.17), we can write

$$
\begin{aligned}
& m_{k}=1 \text { for } 0 \leq \varepsilon<\varepsilon_{A k}, \\
& m_{k}=0 \text { for } \varepsilon_{A k} \leq \varepsilon<1, \\
& n_{k}=1 \text { for } 0 \leq \varepsilon<\varepsilon_{B k}, \\
& n_{k}=0 \text { for } \varepsilon_{B k} \leq \varepsilon<1 .
\end{aligned}
$$

In terms of these two parameters, we can express the per unit time for three intervals:

(a) $0 \leq \varepsilon<\varepsilon_{A k}$ prepulse per unit time: $m_{k}=n_{k}=1$,

(b) $\varepsilon_{A k} \leq \varepsilon<\varepsilon_{B k}$ inside-pulse per unit time: $m_{k}=0, n_{k}=1$,

(c) $\varepsilon_{B k} \leq \varepsilon<1$ postpulse per unit time: $m_{k}=n_{k}=0$.

After doing that, we can use the residua theorem in the modified $Z$-transform to find an analytical closed-form solution

$$
\mathbf{i}_{S}(n, \varepsilon)=\frac{1}{2 \pi j} \oint \mathbf{i}_{S}(z, \varepsilon) z^{n-1} d z .
$$

The solution contains three terms

$$
\mathbf{i}_{S}(n, \varepsilon)=L^{-1}\left\{\mathbf{i}_{S}(s)\right\}=\mathbf{i}_{\mathrm{SS}}(n, \varepsilon)+\mathbf{i}_{\mathrm{SL}}(n, \varepsilon)+\mathbf{i}_{\mathrm{SV}}(n, \varepsilon),
$$

where $L^{-1}$ means the inverse Laplace transform. But this step must be done by means of the mixed $p-z$ approach as mentioned here and demonstrated in [7].

These three fictitious currents are (see (4.10)) as follows:

(i) $\mathbf{i}_{\mathrm{SS}}(n, \varepsilon)$ is a fictitious current from the grid voltage $\mathbf{v}_{S}$,

(ii) $\mathbf{i}_{\mathrm{SL}}(n, \varepsilon)$ is the second fictitious current given by the voltage drop $Z_{F} \mathbf{i}_{L}$,

(iii) $\mathbf{i}_{\mathrm{SV}}(n, \varepsilon)$ is the third fictitious current given by a voltage of the four-switch converter $\mathbf{v}^{*}$.

The solution contains both the steady state and transient components. As our attention here is on the steady states we can obtain a steady state, solution in the closed form for $n \rightarrow \infty$. The current time responses in the steady state for some HPF parameters are presented in the next paragraph. 


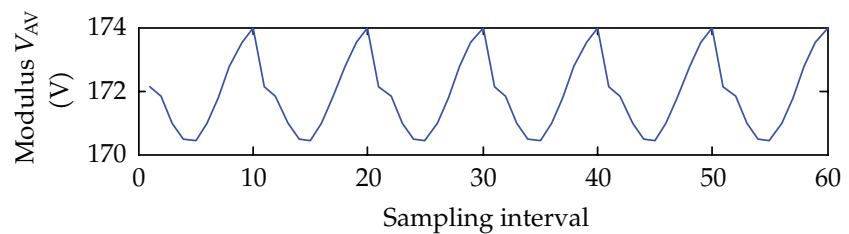

(a)

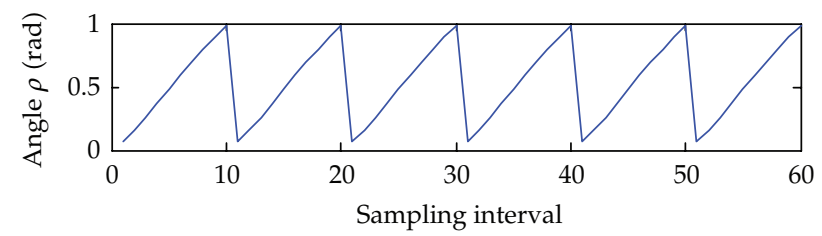

(b)

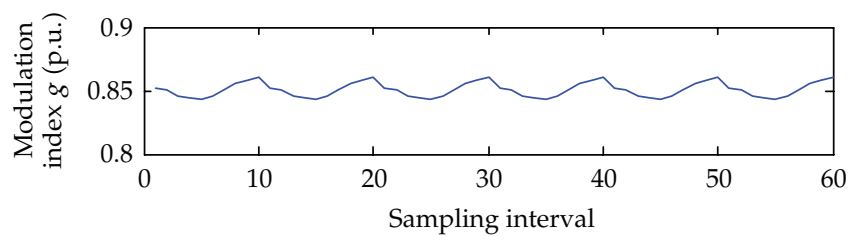

(c)

Figure 5: Time responses of variables $\left|\mathbf{V}_{\mathrm{AV}}\right|, \rho$ and modulation index $g$ within one period of the fundamental frequency.

\section{Simulation}

The presented simulation results were obtained for the following parameters:

$$
\begin{array}{lll}
V_{S}=120 \mathrm{~V}, & V_{\mathrm{dc}}=300 \mathrm{~V}, \quad I_{S}=1.5 \mathrm{~A}, \quad R_{S}=1.4 \Omega, \\
L_{S}=0.01 \mathrm{H}, \quad R_{F}=1.0 \Omega, & L_{F}=0.005 \mathrm{H}, \quad C_{F}=0.000041 \mathrm{~F}, \quad \alpha_{h}=1,
\end{array}
$$

$f S W=3 \mathrm{kHz}$.

The parameters $R_{S}, L_{S}$ involve also the parameters of an autotransformer and an additional resistor used in a laboratory setup for the verification of simulation results.

Figure 5 presents the time responses of the variables $\left|\mathbf{V}_{\mathrm{AV}}\right|, \rho$ and the modulation index $g$ given by (4.9) and (3.10) within one fundamental frequency period.

Figure 6 shows the respective values of the time interval lengths $\Delta \varepsilon_{1}, \Delta \varepsilon_{2}, \Delta \varepsilon_{3}, \Delta \varepsilon_{4}$, and $1 / N_{1}=\Delta \varepsilon_{1}+\Delta \varepsilon_{2}+\Delta \varepsilon_{3}+\Delta \varepsilon_{4}$ calculated in each of 60 sampling intervals from (3.9) within the basic period $T_{1}=1 / 50=0.02 \mathrm{~s}$.

We can see that the magnitude $\mathbf{V}_{\mathrm{AV}}$ and therefore the modulation index $g$ too, as well as the angle $\rho$, are time dependent within the fundamental period $T_{1}$ even in steady states due to the fact that VSC is only two converter-legs (B4) connection and generates a nonsinusoidal voltage $\mathbf{v}^{*}$ for load current harmonics compensation.

Trajectories of three fictitious currents forming overall grid current in complex $\alpha \beta$ plane are shown in Figure 7. 


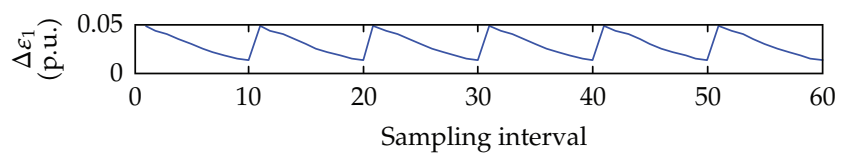

(a)

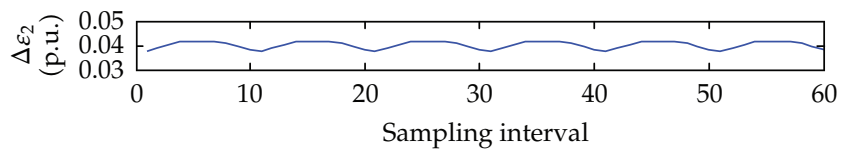

(b)

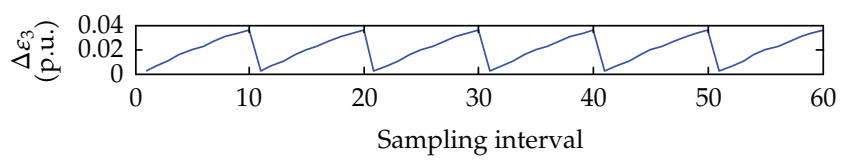

(c)

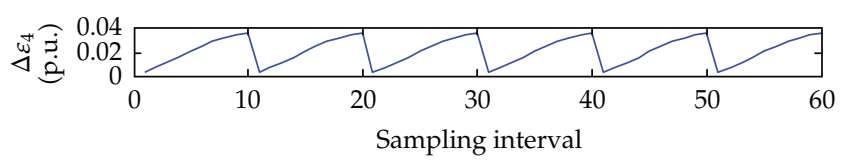

(d)

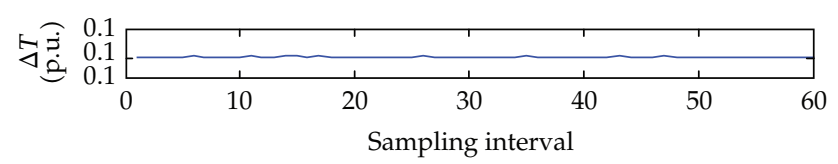

(e)

Figure 6: Values of time interval lengths $\Delta \varepsilon_{1}, \Delta \varepsilon_{2}, \Delta \varepsilon_{3}, \Delta \varepsilon_{4}$, and $1 / N_{1}=\Delta \varepsilon_{1}+\Delta \varepsilon_{2}+\Delta \varepsilon_{3}+\Delta \varepsilon_{4}$ calculated in each of 60 sampling intervals.

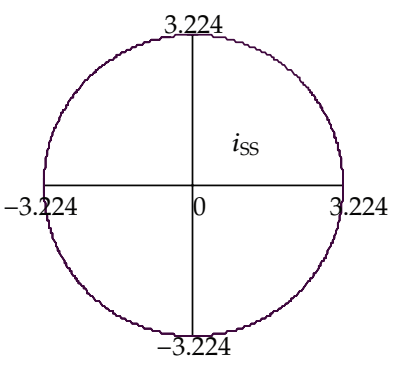

(a)

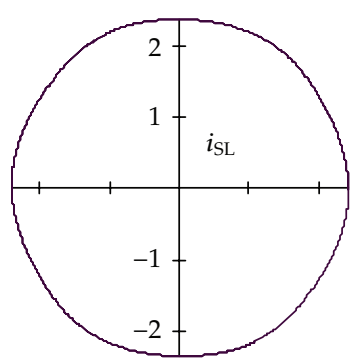

(b)

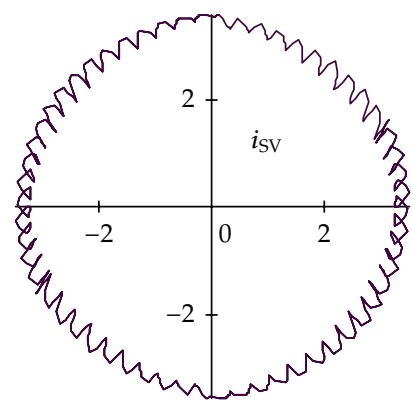

(c)

Figure 7: Trajectories of three fictitious currents forming overall grid current: $i_{\mathrm{SS}}(\mathrm{a}), i_{\mathrm{SL}}(\mathrm{b})$, and $i_{\mathrm{SV}}(\mathrm{c})$.

In Figure 8, we can see the trajectories of the grid overall current and also the phase current waveforms.

In Figure 9, we can see the compensating HPF current. All these results in steady states were calculated by using the closed-form equations and visualized by the program MATHCAD. 


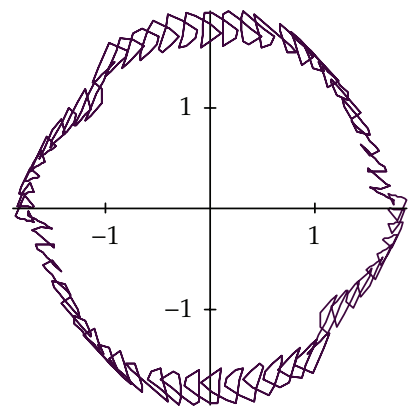

(a)

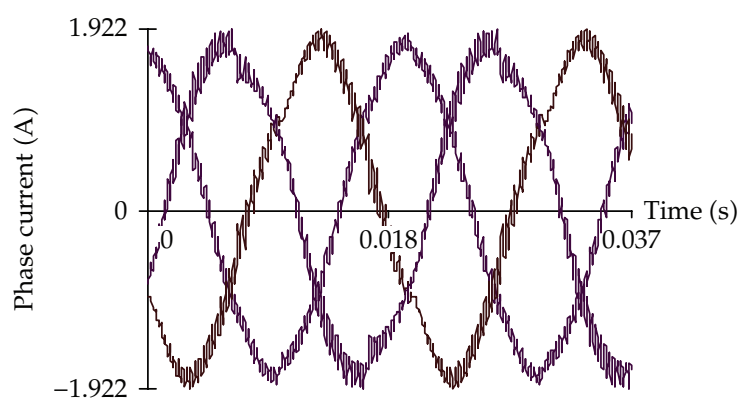

(b)

Figure 8: Space vector of grid current (a) and three phase waveforms (b).

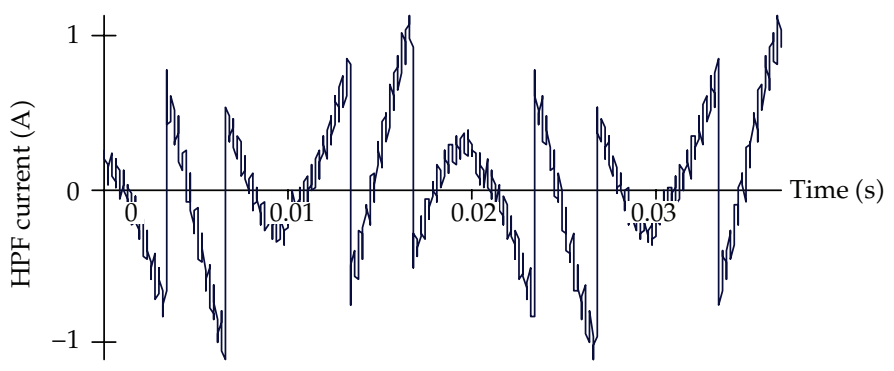

Figure 9: HPF current.

Better results than those presented here can be expected for switching frequencies higher than $3 \mathrm{kHz}$. The switching frequency $3 \mathrm{kHz}$ was selected because of the complexity of the code running in dSPACE 1103 control system used in a laboratory setup for the verification of the simulation results.

\section{Experimental Results}

We verified the proposed method through experiments. The experimental system consists of a three-phase converter, $R_{F}, L_{F}$, and $C_{F}$ passive filter (tuned to the 7 th harmonic), and a nonlinear load (a three-phase uncontrolled rectifier). The setup contains also digital control unit based on the dSPACE control system DS 1103. The converter is an integrated power module MITSUBISHI PS12038 (1200 V, 25 A, $15 \mathrm{kHz}$ at maximum). The parameters of the experimental setup are given in Table 1 .

The grid currents are detected as well as the voltage at the point of common coupling (PCC) and the dc voltage $V_{\mathrm{dc}}$. The input signal is the reference voltage for this dc voltage.

The harmonic current detective control is applied where grid phase currents are transformed into two orthogonal components in the reference frame rotating with the synchronous angular speed $\omega_{1}$. Fluctuating $d q$ components of the source current are obtained by using a high pass filter (HP) of the first order with the cut-off frequency $f_{c}=16 \mathrm{~Hz}$. These components are to be compensated by the HPF.

In order to control the magnitude of the dc voltage $V_{\mathrm{dc}}$, an additional control loop is inserted into the $q$ axis path in the feedback control loop. The reference-compensating 
Table 1: Experiment parameters.

\begin{tabular}{lccc}
\hline Grid and autotransformer & $R_{S}=1.4 \Omega$ & $L_{S}=11.1 \mathrm{mH}$ & \\
Passive filter & $R_{F}=1 \Omega$ & $L_{F}=5 \mathrm{mH}$ & $C_{F}=41 \mu \mathrm{F}$ \\
Active power filter & $C=832 \mu \mathrm{F}$ & $f_{\mathrm{SW} w}=3 \mathrm{kHz}$ & $t_{\mathrm{dead}}=1 \mu \mathrm{S}$ \\
HP signal filter & $f_{c}=16 \mathrm{~Hz}$ & 1 st order & For detection of harmonic currents \\
\hline
\end{tabular}

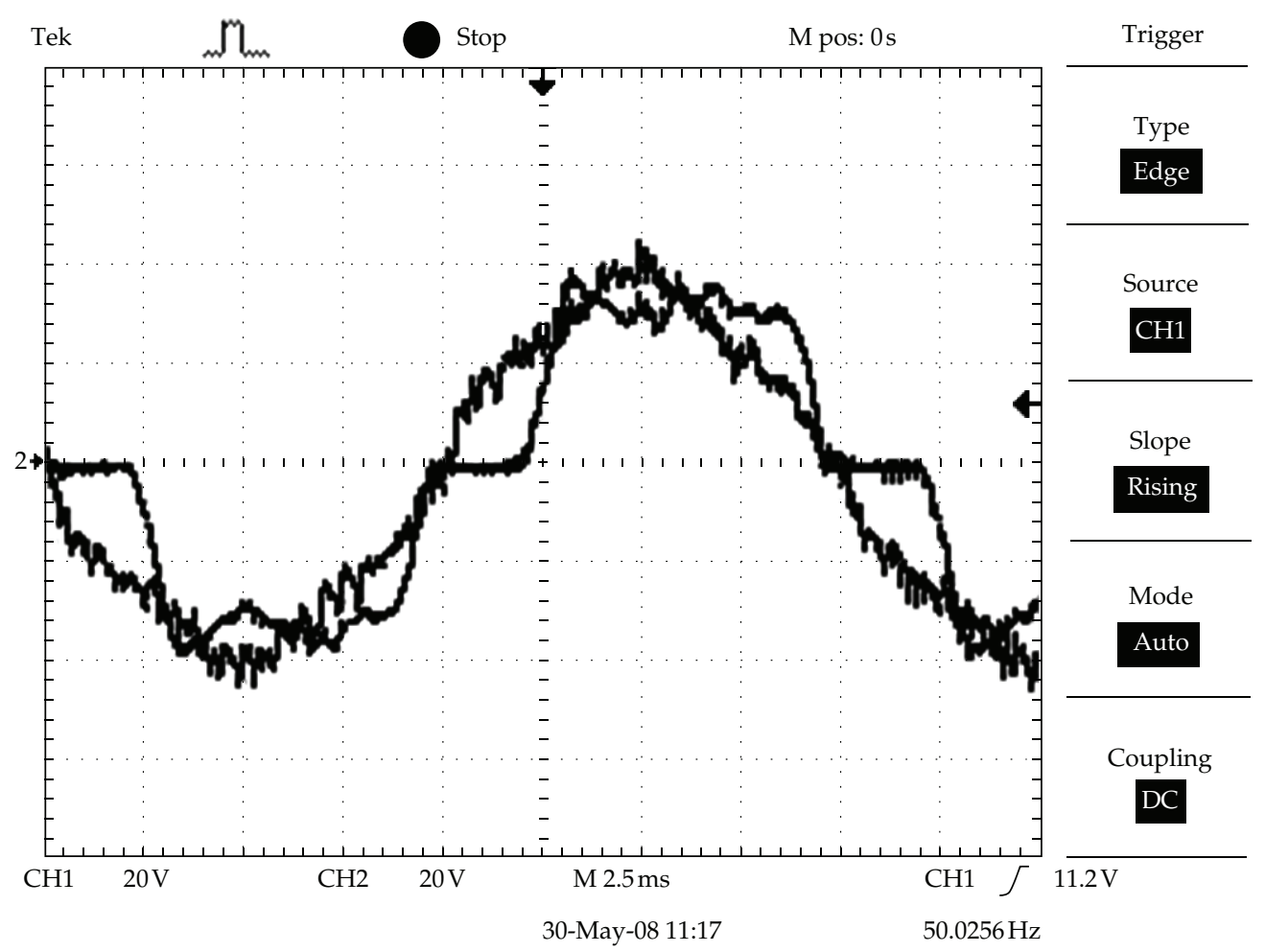

Figure 10: Load and grid phase currents behavior (1 A/div).

current components $i_{\mathrm{Sd}}^{*} i_{\mathrm{Sq}}^{*}$ are then transformed back into the phase reference quantities and multiplied by a feedback control gain. Thus, three reference phase voltages for the HPF are produced which are generated by the feedback control loop.

Figure 10 shows the trajectories of the load and grid phase currents. The control loop of the nonactive power has not been activated here so that a phase shift between the load and source currents is evident due to the capacitor of the HPF passive part.

Figure 11 presents the trajectories of the load and grid phase currents (a), harmonic spectra of the load current (b), and the grid current (c). It is evident that the 5 th, 11th, and 13th current harmonics are suppressed approximately three times, but the 7th harmonic is lowered roughly six times because the HPF passive part is tuned to the 7th harmonic $(350 \mathrm{~Hz})$.

The residual harmonics in the grid current result from a small residual third-order voltage harmonic due to the PWM strategy used and mainly from the fact that the feedback control gain of the feedback control strategy must be limited to guarantee the stability of the control loop. Some specific effects, like the function of a PLL (phase-locked loop) used to synchronize the control algorithm, dead times of switching elements, and so forth, decrease 


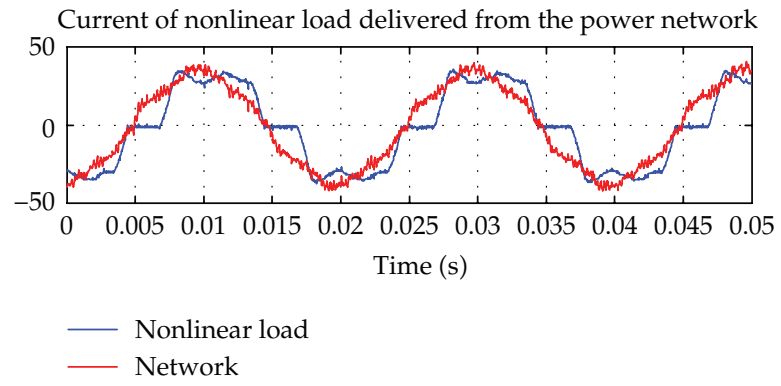

(a)

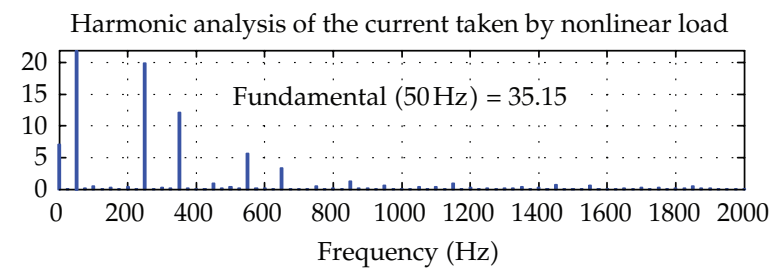

(b)

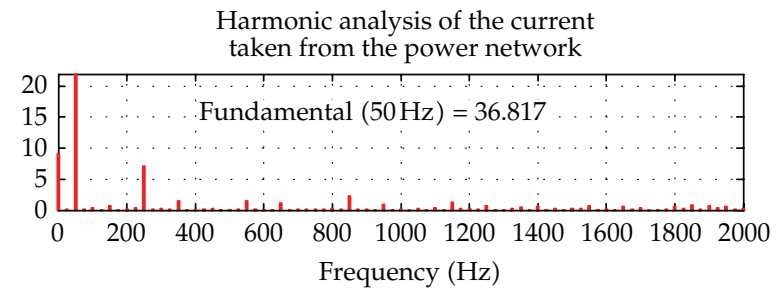

(c)

Figure 11: Trajectories of the load and grid phase currents ((a) $2 \mathrm{~A} / 50 \mathrm{div})$, harmonic spectrum of the load current (b), and the grid current (c).

the efficiency to cancel out the load current harmonics completely. It should be noted as well that the ac voltage at the PCC is far from being very sinusoidal due to the relatively high source impedance $R_{S}, L_{S}$.

It is clear that the analyzed topology can supply a constant reactive power proportional to the value of the condenser $C_{F}$ of the passive part to the grid. The condensers voltage rating is higher than that for a normal (B6) converter because of their additional dc voltage. But this reduced topology saves power electronics-based switching elements and their control and protection circuits. The advantage of the topology and the proposed space vector modulation strategy is that the center of the dc-bus voltage need not be accessible, and the control preserves the same switching symmetry (sixfold) as in the normal (B6) converter. Thus, the power circuitry and controller reconfiguration in case of the transition from B6 to B4 topology due to a fault is easy.

\section{Conclusion}

In this paper, we proposed the analytical model and the original space vector modulation strategy for a new circuit configuration of a hybrid power filter with only four switches and 
not utilizing the center point of the dc-bus voltage. This configuration removes the problems with pulsation of the dc-bus voltage known from the bus-clamped converters. Based on the control algorithm, nearly sinusoidal grid currents with the nonlinear load currents are achieved by the adopted converter. Finally, an original closed formsolution of steady state grid currents, based on the mixed $p-z$ approach, is introduced. The analytical results and experimental tests on the component-minimized three-phase HPF are shown as well.

\section{Acknowledgment}

This paper is dedicated to the memory of Professor Jiři Klíma who passed away on December 2009 at the age of 64.

\section{References}

[1] J. C. Wu, H. L. Jou, Y. T. Feng, W. P. Hsu, M. S. Huang, and W. J. Hou, “Novel circuit topology for three-phase active power filter," IEEE Transactions on Power Delivery, vol. 22, no. 1, pp. 444-449, 2007.

[2] C. Cecati and N. Rotondale, "A double PWM-strategy for improved electric drive reliability," in Proceedings of the International Conference on Power Electronics, Electrical Drives, Automation and Motion (SPEEDAM '02), pp. A2-25-A2-30, 2002.

[3] D. Kastha and B. K. Bose, "Investigation of fault modes of voltage-fed inverter system for induction motor drive," IEEE Transactions on Industry Applications, vol. 30, no. 4, pp. 1028-1038, 1994.

[4] B. R. Lin and T. C. Wei, "Analysis and implementation of a three-phase two-leg neutral point clamped converter based on space vector PWM for power factor correction," IEE Proceedings: Electric Power Applications, vol. 151, no. 1, pp. 38-46, 2004.

[5] J. Klima, J. Skramlik, and V. Valouch, "An analytical modelling of three-phase four-switch PWM rectifier under unbalanced supply conditions," IEEE Transactions on Circuits and Systems II, vol. 54, no. 12, pp. 1155-1159, 2007.

[6] J. Klima, J. Skramlik, and V. Valouch, "Analytical modeling and implementation of a new four-switch hybrid power filter topology," in Proceedings of the International Conference on Renewable Energies and Power Quality (ICREPQ '08), March 2008.

[7] J. Klima, "Mixed p-z approach for time-domain analysis of voltage source inverters with periodic pulsewidth modulation," IEEE Transactions on Circuits and Systems II, vol. 51, no. 10, pp. 529-536, 2004.

[8] J. Klima, "Analytical closed-form solution of a space-vector modulated VSI feeding an induction motor drive," IEEE Transactions on Energy Conversion, vol. 17, no. 2, pp. 191-196, 2002. 


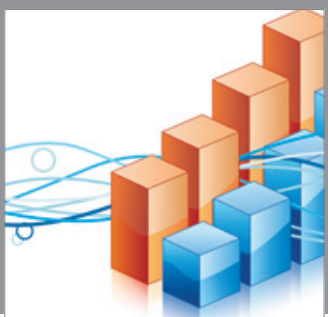

Advances in

Operations Research

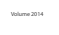

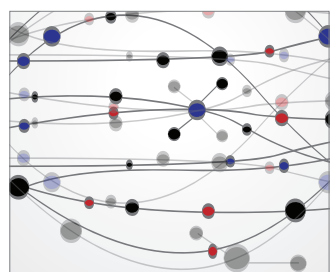

\section{The Scientific} World Journal
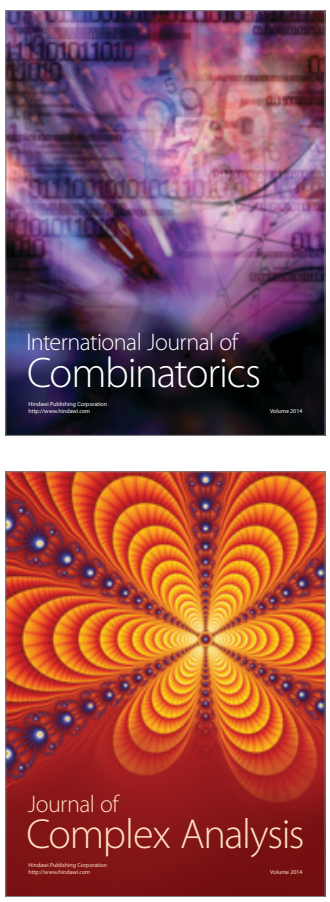

International Journal of

Mathematics and

Mathematical

Sciences
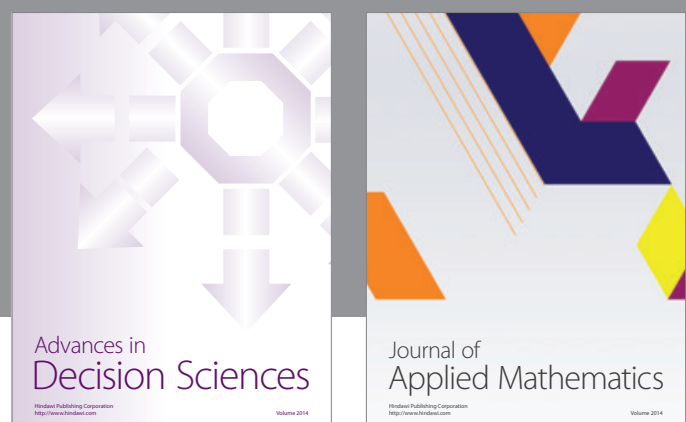

Journal of

Applied Mathematics
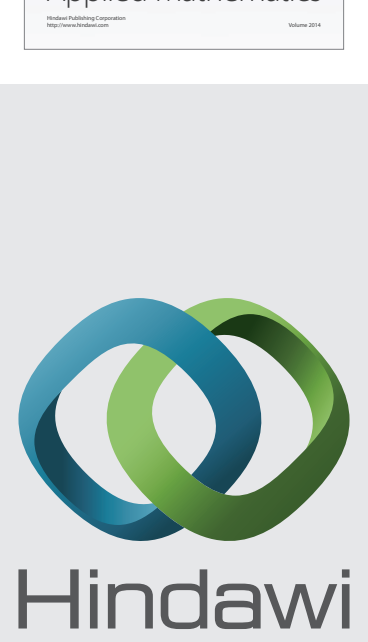

Submit your manuscripts at http://www.hindawi.com
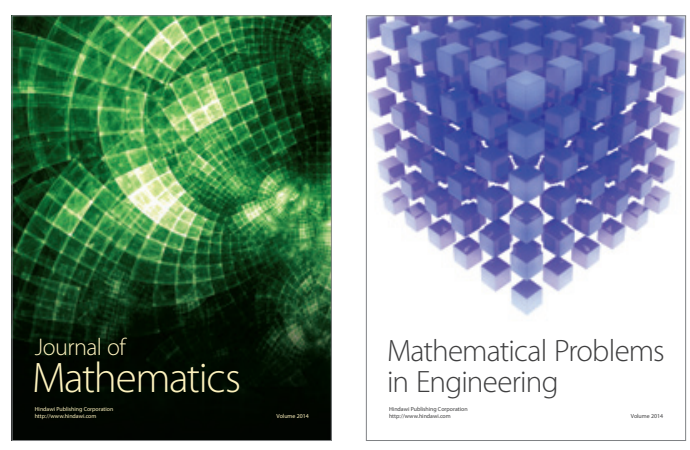

Mathematical Problems in Engineering
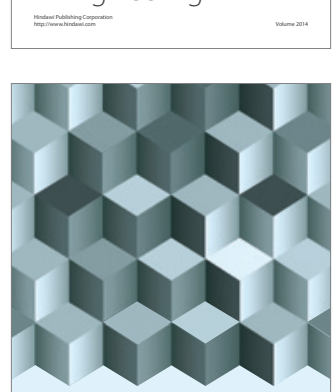

Journal of

Function Spaces
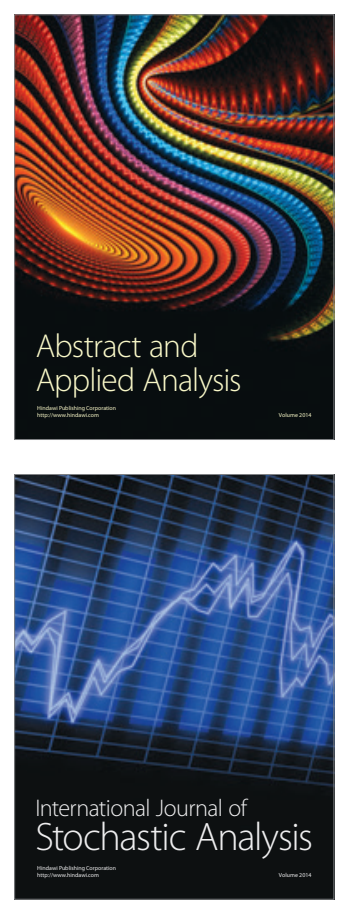

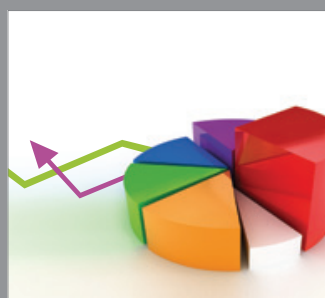

ournal of

Probability and Statistics

Promensencen
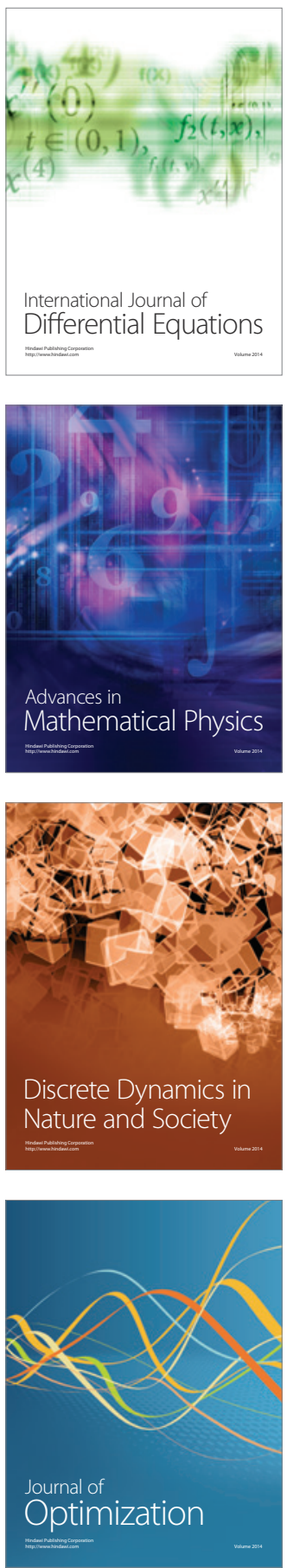